\title{
The disintegrin domain of ADAM17 antagonises fibroblast-carcinoma cell interactions
}

\author{
AHMAD TRAD, MICHEL RIESE, MOHAMMAD SHOMALI, NINA HEDEMAN, \\ TIMO EFFENBERGER, JOACHIM GRÖTZINGER and INKEN LORENZEN
}

Institute of Biochemistry, Christian-Albrechts University of Kiel, D-24118 Kiel, Germany

Received January 4, 2013; Accepted February 12, 2013

DOI: 10.3892/ijo.2013.1864

\begin{abstract}
The malignant phenotype of carcinoma cells depends on their ability to invade into their microenvironment promoting metastasis. Therefore, carcinoma cells overexpress many proteins, including A disintegrin and metalloproteases (ADAMs). ADAM17 is expressed by different cancer cell lines and possesses adhesive as well as enzymatic activities. To address the adhesive properties in tumour progression the recombinantly expressed soluble disintegrin domain of ADAM17 was employed. Fibroblasts and carcinoma cells adhere to the immobilized disintegrin domain. Additionally, the soluble disintegrin domain impaired fibroblast-carcinoma cell interactions and increased the shedding activity of ADAM17. Silencing of ADAM17 in fibroblasts or in carcinoma cells decreases cell-cell interaction between these cells. In summary, our results show that the adhesive properties of ADAM17 are mediated by its disintegrin domain and enables carcinoma cells to interact with their microenvironment.
\end{abstract}

\section{Introduction}

The ability of carcinoma cells to spread to other sites within the body requires different biological processes including modulation of adhesion molecules on the cell surface. ADAMs are known to exert adhesive functions as well as proteolytic activity $(1,2)$, implying a critical role in carcinoma metastasis. ADAMs are type-I transmembrane glycoproteins sharing a common structure $(3,4)$. Starting from the N-terminus ADAM17, like ADAM10, comprises of a signal peptide and a pro-, a catalytic-, a disintegrin- and a membrane-proximal domain, followed by a transmembrane and an intracellular region $(3,5)$. The prodomain is cleaved during maturation in the Golgi apparatus (6). The catalytic domain contains a zinc-binding motif with three conserved histidine residues (HEXGHXXGXXHD) and

Correspondence to: Dr Joachim Grötzinger, Institute of Biochemistry, Christian-Albrechts University of Kiel, Olshausenstr. 40, D-24118 Kiel, Germany

E-mail: jgroetzinger@biochem.uni-kiel.de

Key words: ADAM17, metalloprotease, metastases, disintegrin domain, integrin is responsible for the ectodomain shedding of cell-surface receptors or ligands $(3,6)$. The disintegrin domain is implicated in interactions between ADAMs and integrins, thereby mediating cell adhesion $(7,8)$ and/or inhibiting enzymatic activity $(9,10)$. The membrane-proximal domain has been suggested to be involved in regulatory affairs, dimerisation as well as in substrate recognition (5,11-16). The cytoplasmic region has been shown to interact with proteins containing SH3 domains and may play a role in regulating protease activity as well as intracellular trafficking (17-19).

ADAM17 is highly expressed in various cancer cells $(20,21)$. Indeed, it has been documented that ADAM17 is upregulated in ovarian cancer $(22,23)$, colon carcinoma (24), lung cancer (25) and other types of cancer (26). Furthermore, it was shown for several types of human cancer that shedding of growth factors by ADAM17 is correlated with the pathogenesis of these diseases (27). Interestingly, all these studies focused on the proteolytic function of ADAM17. They considered ADAM17 as the major sheddase of growth factors required for cancer progression and disregarded its adhesive properties. Since ADAM17 is known to interact with $\alpha 5 \beta 1$ integrin (7), we aimed to clarify the role of ADAM17 as an adhesion molecule. Therefore, the disintegrin domain of ADAM17 was expressed as a soluble domain and tested for its ability to bind to fibroblasts/carcinoma cells and to enhance ADAM17 shedding activity. Moreover, the effect of silencing ADAM17 was investigated with respect to fibroblast-carcinoma cell interactions. Taken together, these data show that fibroblast and carcinoma cells adhere to the disintegrin domain of ADAM17 and that ADAM17 is involved in the interaction between carcinoma and fibroblasts.

\section{Materials and methods}

Antibodies. For western blot analysis anti-integrin $\alpha 5$ antibody (sc-10729) was purchased from Santa Cruz Biotechnology (Santa Cruz Biotechnology, Heidelberg, Germany). A300, A300E and A318 are mouse anti-human ADAM17 monoclonal antibodies generated in our laboratory (28). While A300 and A318 bind to disintegrin domain, A300E recognizes the membrane-proximal domain of ADAM17 $(29,30)$.

Cell culture. Mouse embryonic fibroblasts (MEF) and human embryonic kidney 293 cells (HEK-293) were cultured in 
Dulbecco's modified Eagle's medium (DMEM) high-glucose supplemented with $10 \%$ fetal calf serum (FCS), penicillin $(60 \mathrm{mg} / \mathrm{l})$ and streptomycin $(100 \mathrm{mg} / \mathrm{l})$ (PAA Laboratories, Cölbe, Germany) in $5 \% \mathrm{CO}_{2}$ at $37^{\circ} \mathrm{C}$ in a humidified atmosphere. The human carcinoma cell lines (MDA-MB-231, Panc89 and Colo357 from Professor Holger Kalthoff, UKSH Kiel, Germany; NCI-H292 from Dr Anja Baumgart, TU Munich, Germany) were cultured in RPMI-1640 medium supplemented with $10 \%$ FCS, penicillin $(60 \mathrm{mg} / \mathrm{l})$ and streptomycin $(100 \mathrm{mg} / \mathrm{l})$.

Expression and purification of the soluble disintegrin domain of ADAM17. The coding sequence of the human ADAM17 disintegrin domain (amino acid residue 475 to 580) was cloned into pcDNA3.1 zeo (-). Thereby the signal sequence of the human IL-6R, followed by a His- and a myc-tag was placed in front of the disintegrin domain. HEK-293 cells were transfected using TurboFect (Fermentas, St. Leon-Rot, Germany) according to the manufacturer's instruction and stably transfected cells were selected. The supernatant of these cells was harvested and the disintegrin domain purified by its N-terminal His-tag using a HisTrap column (GE Healthcare, Freiburg, Germany) followed by size-exclusion chromatography (Superdex 200 column, GE Healthcare). The identity and purification was confirmed by SDS-PAGE and western blot analysis.

Cell-adhesion assays. Microtiter tissue culture plates (Sarstedt, Nümbrecht, Germany) were coated with indicated concentrations of the disintegrin domain (28) in coating buffer $(0.5 \mathrm{M}$ carbonate-bicarbonate buffer, $\mathrm{pH} 9.6)$ at $4^{\circ} \mathrm{C}$ overnight. Plates were blocked with heat-denatured BSA $\left(85^{\circ} \mathrm{C}\right.$ for $10 \mathrm{~min}$, $1 \% \mathrm{BSA}$ in $\mathrm{Ca}^{2+} / \mathrm{Mg}^{2+}$-free $\mathrm{PBS}$ ) for $1 \mathrm{~h}$ at room temperature. In parallel, MEF and carcinoma cells were detached with trypsin. The cells were resuspended in complete medium (DMEM, 10\% FCS, $0.1 \%$ BSA, $1 \mathrm{mM} \mathrm{CaCl}_{2}, 1 \mathrm{mM} \mathrm{MnCl} 2,1 \mathrm{mM} \mathrm{MgCl}_{2}$ ). Plates were washed, $3 \times 10^{4}$ cells/well were added and incubated for $1 \mathrm{~h}$ at $37^{\circ} \mathrm{C}$. In case of the competition assays, cells were first incubated for $1 \mathrm{~h}$ at $37^{\circ} \mathrm{C}$ with the soluble disintegrin domain, washed and then added to the plates coated with the disintegrin domain. Non-adhering cells were removed by washing with complete medium, while attached cells were quantified using titer blue reagent (Promega, Mannheim, Germany). Adhesion was expressed as percentage relative to FCS control, which was set arbitrarily to $100 \%$ (31).

Cell-cell adhesion assays. The adhesion assay of carcinoma cells to fibroblasts has been previously described (31). Briefly, carcinoma cells were labeled with $10 \mu \mathrm{M}$ Calcein AM (Invitrogen, Darmstadt, Germany) for $1 \mathrm{~h}$ at $37^{\circ} \mathrm{C}$. Labeled cells $\left(3 \times 10^{4}\right)$ were washed three times with wash solution (HBSS, $1 \mathrm{mM} \mathrm{MnCl} 2,1 \mathrm{mM} \mathrm{MgCl} 2,1 \mathrm{mM} \mathrm{CaCl}_{2}$ ), incubated with or without disintegrin domain for $1 \mathrm{~h}$ at $37^{\circ} \mathrm{C}$ and seeded onto $80 \%$ confluent monolayers of MEF in 96 -well plates for $30 \mathrm{~min}$. In a parallel experiment, anti-integrin $\beta 1$ inhibitory antibody (clone P4C10, Millipore, Schwalbach, Germany) was added to carcinoma cells instead of disintegrin domain. Not adhered cells were removed by washing, while attached cells were lysed by adding $20 \mu \mathrm{l}$ of $10 \%$ Triton X-100 and qualified by measurement of their fluorescence $(480 \mathrm{~nm} / 530 \mathrm{~nm})$ using fluorescence reader
(BioTek, Bad Friedrichshall, Germany). Statistical analysis was performed with Student's t-test (http://www.physics.csbsju.edu/ stats/t-test_bulk_form.html).

Generation of DN-ADAM17 stable transfect MDA-MB-231 cells. The dominant negative (DN)-ADAM17 is a construct of human ADAM17 that lacks the catalytic domain (32). The human ADAM17 sequence (starting from amino acid residue 475) is placed directly behind the signal peptide of human interleukin-6 in pcDNA3.1 expressing a gentamycin resistance (32). MDA-MB-231 cells were transfected with the plasmid using TurboFect transfection reagent (Fermentas) according to the manufacturer's protocol. Stable transfected clones were selected and grown in medium containing G418 (PAA Laboratories). The expression of DN-ADAM17 was confirmed by flow cytometry and western blot analysis and clone 5 (DN-5) was chosen for further experiments. The DN-ADAM17 construct contains a C-terminal His- and myc-tag.

Co-immunoprecipitation. Stably transfected MDA-MB231 cells (DN-5) were cultured in RPMI-1640 medium, harvested, lysed in $250 \mu 1$ lysis buffer [ $20 \mathrm{mM}$ Tris- $\mathrm{HCl}$ $\mathrm{pH}$ 7.6, $150 \mathrm{mM} \mathrm{NaCl}, 2 \mathrm{mM}$ EDTA and 'complete' protease inhibitor (Roche Applied Science, Mannheim, Germany)] and centrifuged for $15 \mathrm{~min}$ at $14,000 \mathrm{rpm}$. Cell lysate $(100 \mu \mathrm{l}$ ) was mixed with $50 \mu \mathrm{l}$ of protein-G agarose (Thermo Scientific-Pierce, Bonn, Germany) and $2.5 \mu \mathrm{g}$ anti- $\alpha 5 \beta 1$ integrin antibodies (NKI-SAM-1, TS2/16; Biozol, Eching, Germany) or $5 \mu \mathrm{g}$ anti-ADAM17 A300E (28). As control, $100 \mu 1$ of cell lysate was mixed with $50 \mu 1$ of protein-G agarose without antibodies. The samples were incubated $2 \mathrm{~h}$ at $4^{\circ} \mathrm{C}$ by gentle agitation, washed five-times with $1,000 \mu 1$ of lysis buffer containing $1 \mathrm{mM} \mathrm{CaCl}_{2}$ and prepared for western blot analysis. To detect the immunoprecipitated proteins, $20 \mu \mathrm{l}$ SDS denatured protein cell lysate was separated on a $12.5 \%$ polyacrylamide gel. After separation by SDS-PAGE, proteins were transferred onto a polyvinylidene-fluoride (PVDF) membrane (GE Healthcare). The PVDF membrane was blocked with $5 \%$ milk powder in TBS-T $(10 \mathrm{mM}$ Tris- $\mathrm{HCl}$, $\mathrm{pH} 7.6,150 \mathrm{mM} \mathrm{NaCl}, 0.1 \%$ Tween-20) for $2 \mathrm{~h}$ and then incubated overnight at $4^{\circ} \mathrm{C}$ with rabbit anti-myc tag $(1 / 1,000$; Cell Signaling, Frankfurt, Germany) or with anti- $\alpha 5$ integrin antibody (sc-10729, Santa Cruz Biotechnology) in 1\% milk powder. After washing, the blots were incubated for $1 \mathrm{~h}$ with an anti-rabbit IgG POD conjugate and visualized by chemiluminescence using an ECL kit (GE Healthcare).

ELISA. Microtiter plates (Nunc, Denmark) were coated with $50 \mu \mathrm{l}(10 \mu \mathrm{g} / \mathrm{ml}$ ) per well of human purified $\alpha 5 \beta 1$ integrin (gift from Dr Humphries, University of Manchester, Manchester, $\mathrm{UK}$ ) in coating buffer (PBS, $1 \mathrm{mM} \mathrm{MnCl}$ ) and incubated at $4^{\circ} \mathrm{C}$ overnight. Then the plates were washed with washing solution (TBS, $1 \mathrm{mM} \mathrm{MnCl}{ }_{2}, 0.1 \%$ BSA) and blocked with 5\% BSA in TBS for $3 \mathrm{~h}$ at room temperature. After washing, $50 \mu \mathrm{l}$ of recombinant disintegrin domain $(80 \mu \mathrm{g} / \mathrm{ml})$ plus $50 \mu \mathrm{l}$ of mouse anti-disintegrin monoclonal antibodies (A300, A318; $20 \mu \mathrm{g} / \mathrm{ml}$ ) (28) or anti-His-tag antibody (1/500; Cell Signaling) were added and incubated for $3 \mathrm{~h}$ at room temperature. Blank control was performed with PBS. The plates were washed with washing solution and the antibodies were detected 

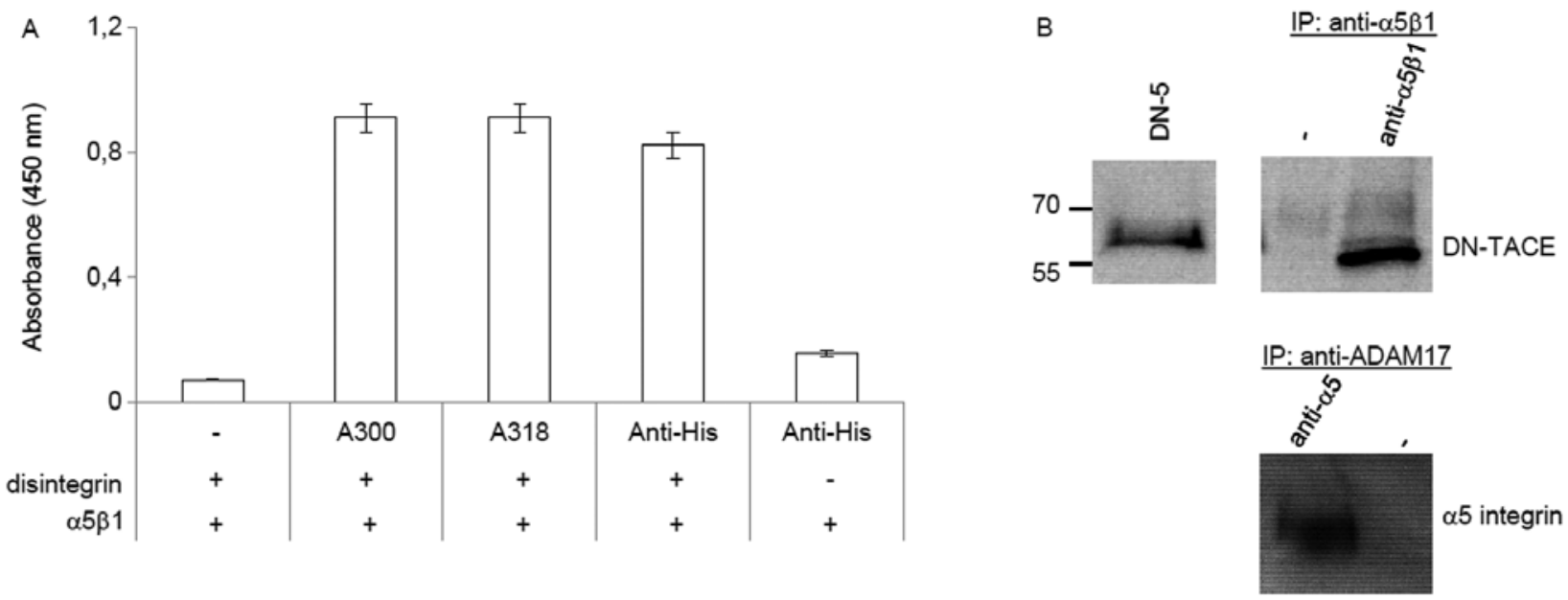

Figure 1. Direct interaction of the disintegrin domain of ADAM17 and $\alpha 5 \beta 1$ integrin. For ELISA experiments, the plates were coated with purified $\alpha 5 \beta 1$ integrin $(10 \mu \mathrm{g} / \mathrm{ml})$. Afterwards $40 \mu \mathrm{g} / \mathrm{ml}$ disintegrin domain was added and its binding detected by anti-disintegrin domain (A300, A318) or anti-His antibodies and anti-mouse-HRP antibodies. (B) Co-immunoprecipitation of DN-ADAM17 by $\alpha 5 \beta 1$ integrin. DN-ADAM17 stably transfected MDA-MB-231 cells (DN-5) were lysed and the presence of DN-ADAM17 was confirmed by western blot analysis using anti-myc tag (left panel). One half of the cell lysate $\alpha 5 \beta 1$ integrin was precipitated using anti- $\alpha 5$ (NKI-SAM-1) and anti- $\beta 1$ (TS2/16) antibodies, as control the other half was treated the same but without antibody (-). Afterwards the precipitates were analyzed by western blotting using anti-myc tag (right panel). In the lower panel, cell lysates of DN-5 were immunoprecipitated with ADAM17 antibody (A300E) and probed for $\alpha 5$ integrin (sc-10729) by western blot analysis. Immunoprecipitation without anti-ADAM17 antibody served as negative control.

by anti-mouse IgG-POD conjugate (Southern Biotech, Birmingham, AL, USA) diluted 1/3,000 in washing buffer. After washing, $80 \mu \mathrm{l}$ of BM blue POD substrate (Roche Applied Science) was added to each well for $10 \mathrm{~min}$. Then, the reaction was stopped by adding $80 \mu \mathrm{l}$ of $0.8 \mathrm{M} \mathrm{H}_{2} \mathrm{SO}_{4}$. Measurement of the optical density (OD) was performed at $450 \mathrm{~nm} / 540 \mathrm{~nm}$ on a plate reader (Tecan, Männedorf, Switzerland).

Flow cytometry (FACS). Cells $\left(5 \times 10^{5}\right)$ were washed twice in FACS solution (PBS, $0.05 \% \mathrm{NaN}_{3}$ ) and stained for $1 \mathrm{~h}$ at $4^{\circ} \mathrm{C}$ with $2 \mu \mathrm{g} / \mathrm{ml}$ of anti-ADAM17 (A300E) antibody directed against the membrane-proximal domain of the enzyme (28). After washing, $2 \mu \mathrm{l}$ of allophycocyanin-conjugated goat anti-mouse Ig (Jackson ImmunoResearch Laboratories, West Grove, PA, USA) was added. The cells were then incubated for $1 \mathrm{~h}$ at $4^{\circ} \mathrm{C}$ and analyzed with a FACScan flow cytometer (BD Biosciences, USA). Cell populations of interest were gated and analyzed using BD FACSDiva software.

Shedding of amphiregulin into the cell culture medium. MDA-MB-231 cells were seeded on 24-well plate (70-80\% confluence) and incubated overnight at $37^{\circ} \mathrm{C}$ in RPMI medium containing $10 \%$ FCS. The soluble disintegrin domain of ADAM17 was added to a final concentration of $20 \mu \mathrm{g} / \mathrm{ml}$ in serum-free medium and incubated for $8 \mathrm{~h}$. The matrix metalloprotease inhibitor, marimastat (MM), was used at final concentration of $3 \mu \mathrm{M}$. The supernatant was collected and the levels amphiregulin were determined using a sandwich ELISA (DuoSet Kit; R\&D Systems, Minneapolis, MN, USA) in accordance with the manufacturer's instructions.

\section{Results}

The disintegrin domain of ADAM17 interacts directly with integrin $\alpha 5 \beta 1$. A previous study has shown that ADAM17 interacts with $\alpha 5 \beta 1$ integrin (7). To verify the specificity of this interaction, we performed an ELISA with the purified disintegrin domain of ADAM17 and the $\alpha 5 \beta 1$ integrin (Fig. 1A). Binding of the disintegrin domain to the purified $\alpha 5 \beta 1$ integrin was detected by monoclonal mouse anti-disintegrin domain antibodies (A300, A318) (28) and confirmed by an anti-His antibody. The ELISA data demonstrate that the disintegrin domain and the $\alpha 5 \beta 1$ integrin interact directly. Co-immunoprecipitation was used as second approach to confirm the interaction between the disintegrin domain and the $\alpha 5 \beta 1$ integrin. Thereby, DN-ADAM17 was detectable in cell lysates from MDA-MB-231 cells stably transfected with the ADAM17 deletion mutant and could be co-immunoprecipitated using anti- $\alpha 5 \beta 1$ antibodies (Fig. 1B). In addition, $\alpha 5$ integrin could be detected after precipitation with anti-ADAM17 antibody (A300E) from cell lysate of DN-ADAM17 stable transfected MDA-MB-231 cells (Fig. 1B). Thus, the disintegrin domain of ADAM17 binds to the $\alpha 5 \beta 1$ integrin.

Fibroblast cells adhere to the disintegrin domain of ADAM17. To examine the adhesive properties of ADAM17, we performed cell adhesion assays using fibroblasts and the disintegrin domain coated on cell culture plates (Fig. 2). Fibroblasts were chosen because they express a wide range of different integrins including $\alpha 5 \beta 1$ (33). The amount of fibroblasts bound to the plates coated with FCS was considered as a positive control and set to $100 \%$. A coating-concentration of $50 \mu \mathrm{g} / \mathrm{ml}$ disintegrin domain leads to a maximal adhesion of MEF cells (Fig. 2A). Further increase in the concentration of the disintegrin domain did not improve binding of the MEFs (data not show). Also human fibroblasts IRM.90 bind to the disintegrin domain of ADAM17 in concentration-dependent manner (Fig. 2B).

Carcinoma cells express ADAM17 and adhere to the disintegrin domain. FACS (Fig. 3A) and western blot (Fig. 3B) 

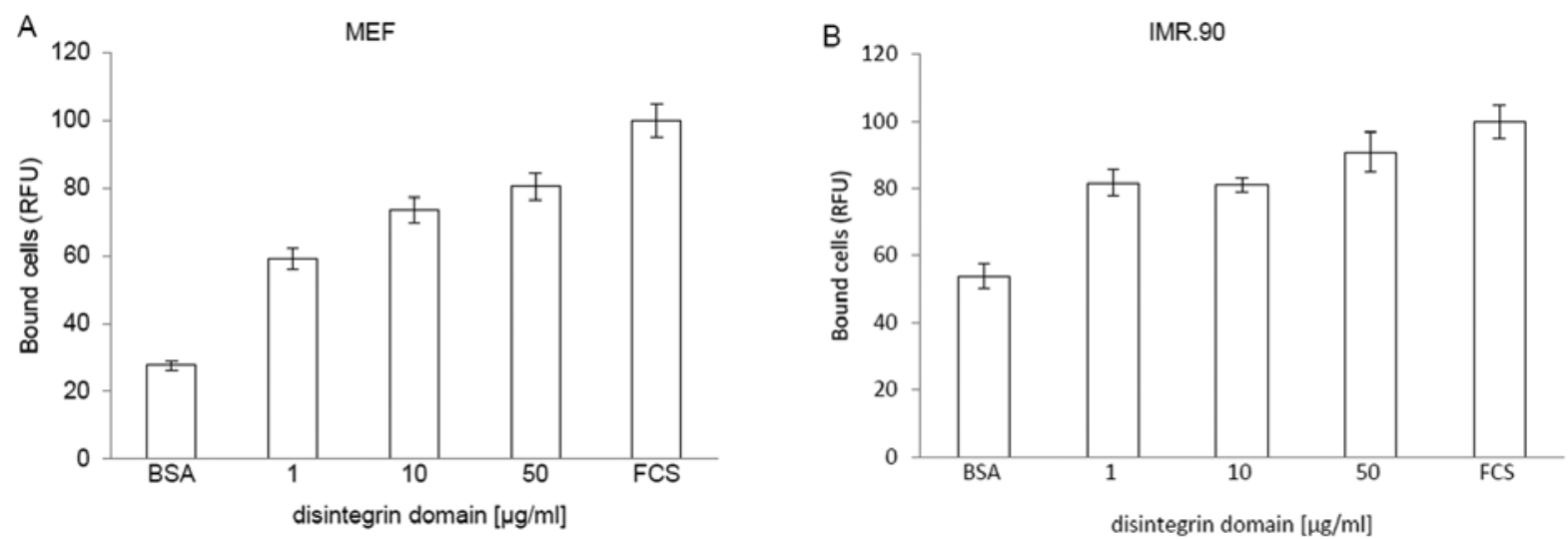

Figure 2. Fibroblasts adhere to disintegrin domain of ADAM17. Cell culture microtiter plates were coated with the indicated concentration of His-tagged disintegrin domain of ADAM17. BSA and FCS were used as negative and positive control, respectively. (A) MEF and (B) IMR.90 cells were added and incubated for $1 \mathrm{~h}$ The attached fibroblasts were quantified using titer blue reagent. Cells number adhered on FCS coated plates was arbitrarily set as 100\%. Bars represent mean \pm SD of the relative fluorescence units of three independent experiments.

A

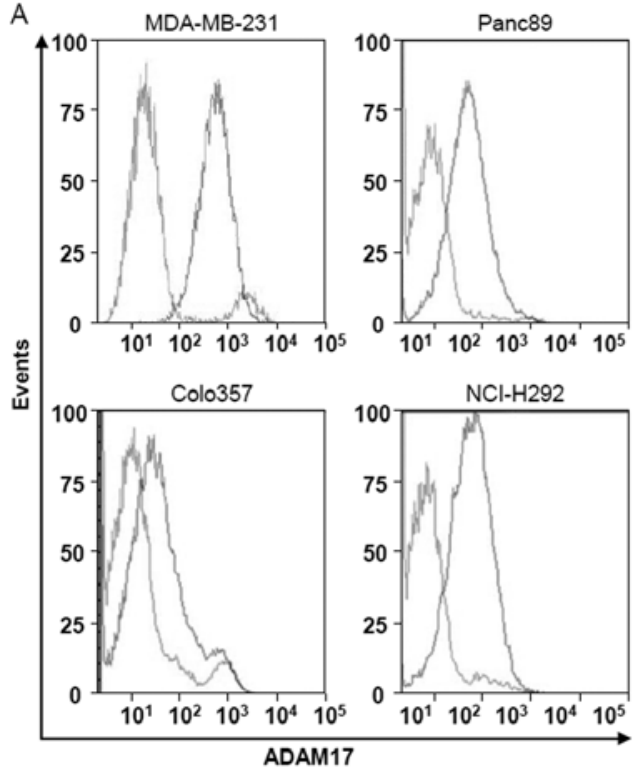

C

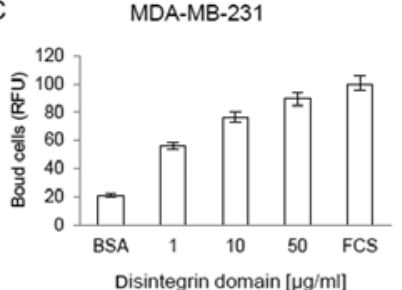

Colo357

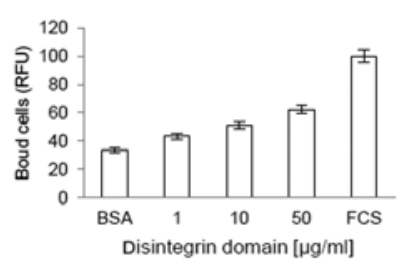

Panc89

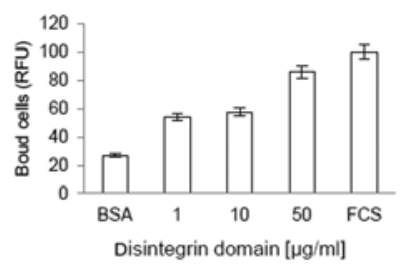

$\mathrm{NCl}-\mathrm{H} 292$

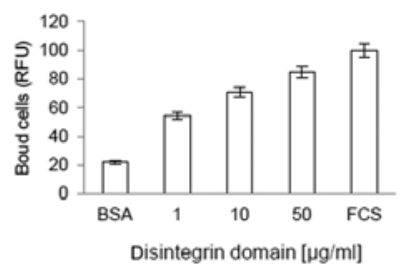

D
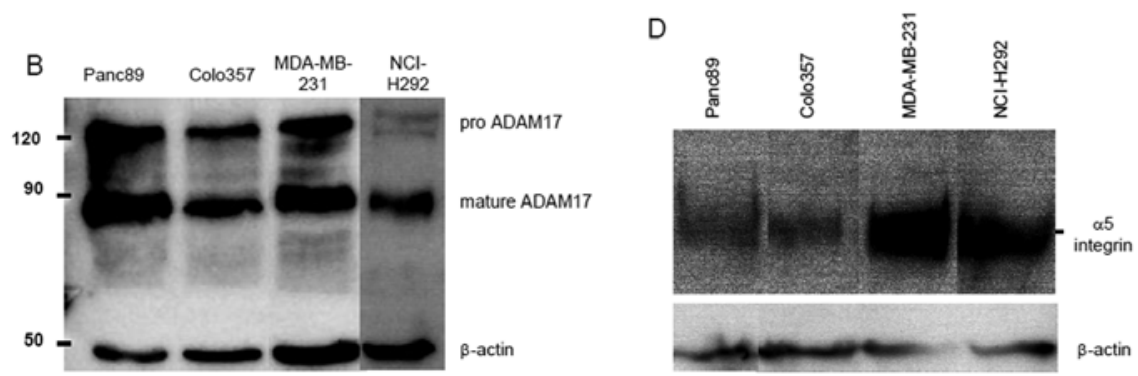

Figure 3. Various carcinoma cells adhere to disintegrin domain of ADAM17. (A) The surface expression of ADAM17 of carcinoma cells was analyzed by flow cytometry (left curve, isotype; right curve, anti-ADAM17 antibody, A300E). Western blot analysis of (B) ADAM17 and (D) $\alpha 5$ integrin expression of carcinoma cells using the anti-ADAM17 A300 and anti- $\alpha 5$ integrin antibody, respectively. (C) For the cell attachment assay cell culture microtiter plates were coated with the indicated concentration of recombinant soluble disintegrin domain of ADAM17. As negative and positive control BSA and FCS were used. The indicated cells were seeded into coated wells and incubated for $1 \mathrm{~h}$. The attached carcinoma cells were quantified using titer blue reagent. Percentage of cell adhesion was determined setting the number adhered to FCS coated cells set to $100 \%$. Bars represent mean \pm SD of the relative fluorescence units of three independent experiments.

analysis demonstrate that carcinoma cells express high levels of ADAM17. This led to the assumption that ADAM17 may be involved in the interactions between fibroblast and carci- noma cells and raised the question, whether upregulation of ADAM17 in carcinoma cells increases the cell-cell interactions between fibroblasts and carcinoma cells. To address this ques- 

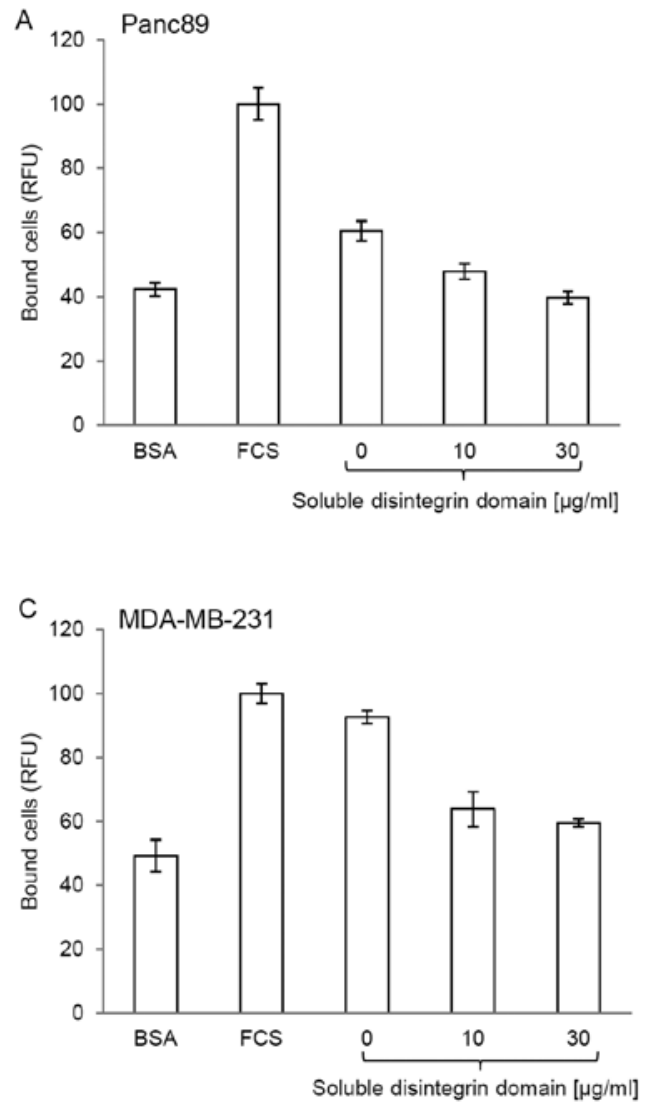

tion two assays were performed. First, we analyzed the binding of carcinoma cells to the immobilized disintegrin domain. Secondly, the interaction between fibroblast and carcinoma cells expressing different level of ADAM17 was evaluated.

Fig. 3C shows that various carcinoma cells (MDA-MB-231, Colo357, Panc89, NCl-H292) bind in a dose-dependent manner to solid phase coupled disintegrin domain. In case of three cells lines (MDA-MB-231, Panc89, NCl-H292) the maximal binding reaches $60 \%$ at a coating-concentration of $50 \mu \mathrm{g} / \mathrm{ml}$ disintegrin domain, in comparison to the positive control coated with FCS; while only $20 \%$ of Colo357 cells bind to the disintegrin domain coated surface. This difference correlates to some extent with the expression level of $\alpha 5$ integrin of these cells (Fig. 3D).

To prove that carcinoma cells and the disintegrin domain interact specifically and directly, we performed a competition assay using soluble and immobilized disintegrin domain. Pretreatment of Panc89 (Fig. 4A), IMR.90 (Fig. 4B) and MDA-MB-231 (Fig. 4C) cells with the soluble disintegrin domain significantly reduced the binding of these cells to the disintegrin domain coated solid phase. A pretreatment with $30 \mu \mathrm{g} / \mathrm{ml}$ of soluble disintegrin domain results in all three cell lines to a decrease in adhesion down to the level of the negative control (plates coated with BSA). In summary, these results indicate that carcinoma cells express $\alpha 5$ integrin and adhere to the disintegrin domain of ADAM17.

ADAM17 mediates fibroblast-carcinoma cell interactions. To evaluate the role of the disintegrin domain at a cellular level, cell-cell interaction assays between fibroblasts and

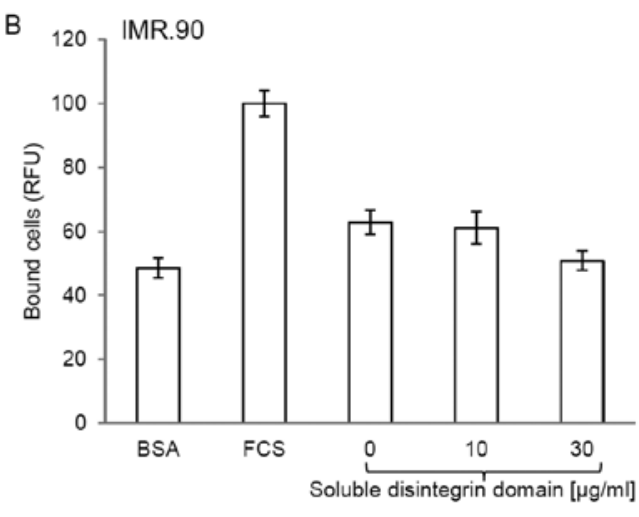

Figure 4. The soluble and immobilized disintegrin domain competes for binding to carcinoma cells. Cell culture microtiter plates were coated with $10 \mu \mathrm{g} / \mathrm{ml}$ of soluble disintegrin domain of ADAM17. BSA and FCS were used as negative and positive control, respectively. (A) Panc89, (B) IMR.90 and (C) MDA-MB-231 cells were incubated with the indicated concentration of soluble disintegrin domain for $1 \mathrm{~h}$ and then added to coated plates. The attached cells were quantified using titer blue reagent. Percentage of adhesion determined on FCS, which was arbitrarily set as $100 \%$. Bars represent mean \pm SD of the relative fluorescence units of three independent experiments.

carcinoma cells in the presence or absence of the disintegrin domain of ADAM17 were performed. In these experiments, the MDA-MB-231 (Fig. 5A), NCI-H292 cells (Fig. 5B) and Panc89 (Fig. 5C) were labeled with Calcein AM, treated with different concentrations of the soluble disintegrin domain and seeded on top of confluent MEF monolayers. The MDA-MB-231, NCI-H292 and Panc89 cells attached to MEFs in the absence of the soluble disintegrin domain. Pretreatment of MDA-MB-231, NCI-H292 and Panc89 cells with the soluble disintegrin domain significantly reduced the interactions between carcinoma and murine fibroblast cells. Similar interactions were observed between the carcinoma and human fibroblasts IMR.90 (Fig. 5D-F). Thus, ADAM17 mediates cell-cell interactions, which can be inhibited by the soluble disintegrin domain.

Silencing of ADAM17 reduces fibroblast-carcinoma cell interaction. In order to evaluate further the adhesive property of the disintegrin domain, we performed cell-cell interaction assays using ADAM17 deficient cells. Whereas ADAM17 deficient cells (ADAM17 ${ }^{\text {ex/ex }} \mathrm{MEFs}$ ) were derived from ADAM17 ${ }^{\text {ex/ex }}$ mice (34), ADAM17 silencing in NCI-H292 cells was achieved using an ADAM17 shRNA (35). ADAM17 ${ }^{\text {ex/ex }}$ mice were generated by insertion of new exon within the gene of ADAM17. This new exon starts with an in-frame translational stop codon. Homozygous ADAM17 ex/ex mice were viable and showed a dramatic loss of ADAM17 protein in all cell types (34). In line with the above results, the amount of ADAM17 knockdown NCI-H292 (-) cells attaching to wt-MEF (+) was significantly reduced as compared to 

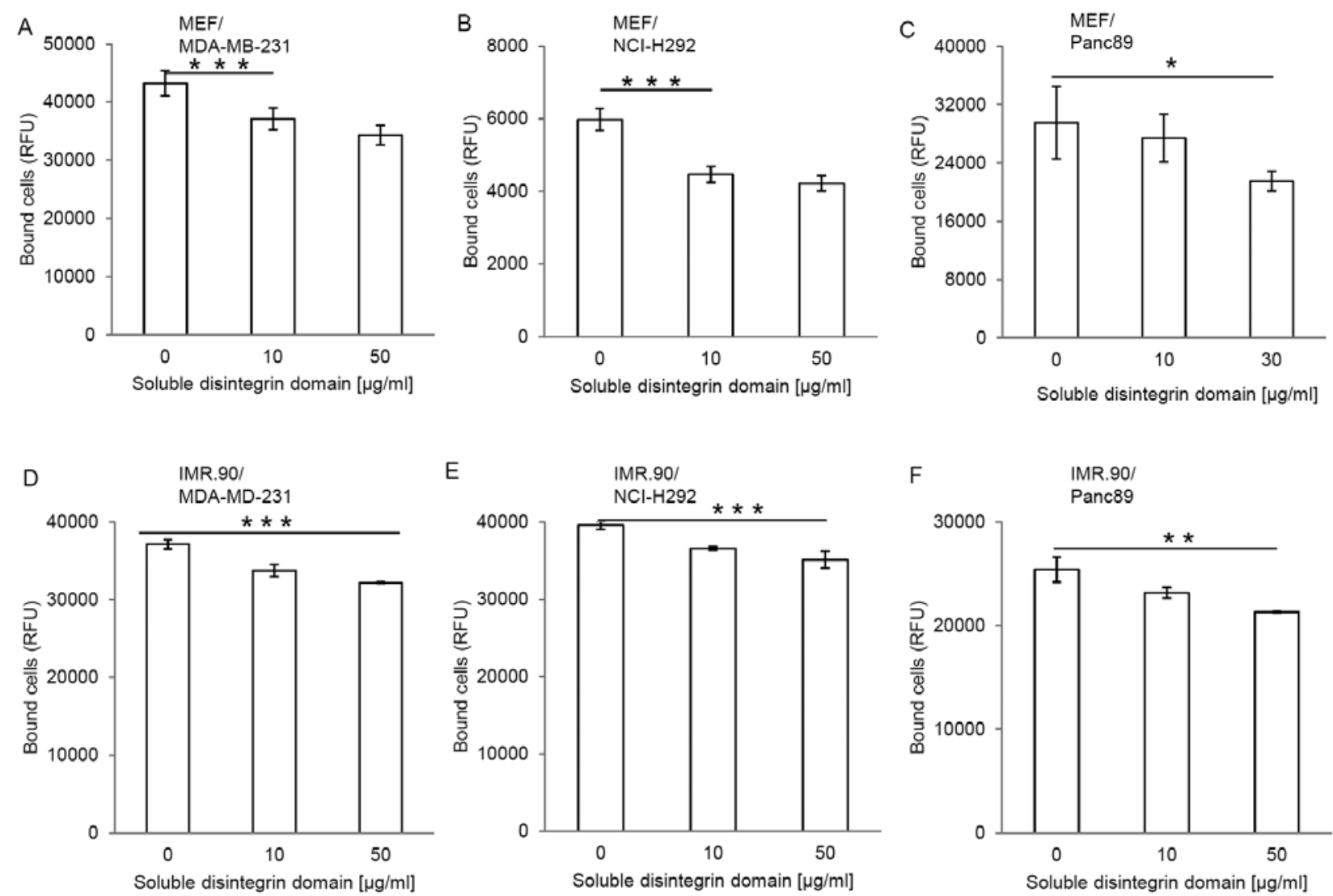

Figure 5. The soluble disintegrin domain of ADAM17 antagonizes the fibroblast-carcinoma cell interactions. MDA-MB-231, NCI-H292 and Panc89 cells were labeled with Calcein AM. Labeled cells were pre-incubated with indicated concentration of soluble disintegrin domain and seeded with (A-C) MEF or (D-F) IMR. 90 cells. After $30 \mathrm{~min}$ at $37^{\circ} \mathrm{C}$, the attached cells were quantified by their fluorescence intensity. Bars represent mean $\pm \mathrm{SD}$ of the relative fluorescence units of three independent experiments. Asterisks indicate significant differences from the respective control values. ${ }^{* *} \mathrm{p}<0.001,{ }^{* * *} \mathrm{p}<0.0001$.

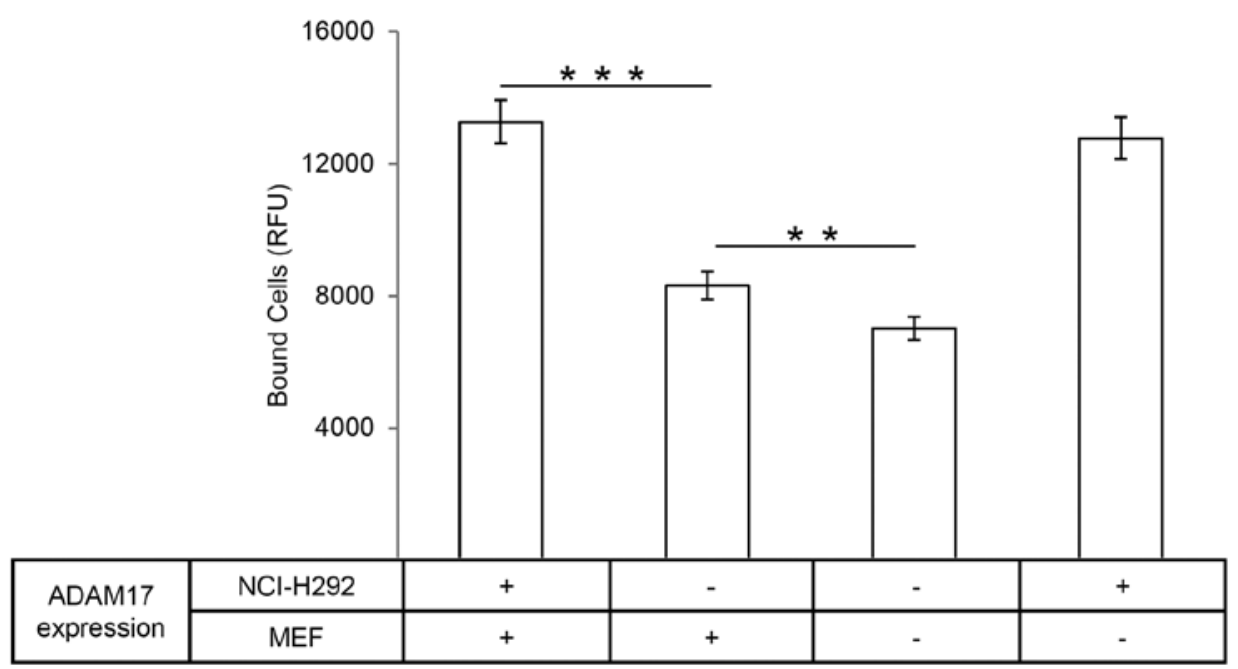

Figure 6. Silencing of ADAM17 expression decreases the fibroblast-carcinoma cell interactions. (A) Wild-type (+) and ADAM17 knockdown (-) NCI-H292 cells were labeled with Calcein AM seeded with wild-type (+) or ADAM17 ${ }^{\text {ex/ex }}(-)$ MEF cells. After $30 \mathrm{~min}$ at $37^{\circ} \mathrm{C}$, the attached cells were quantified by their fluorescence intensity. Bars represent means \pm SD of three independent experiments. Asterisks indicate significant differences from the respective control values $\left({ }^{* *} \mathrm{p}<0.001,{ }^{* * *} \mathrm{p}<0.0001\right)$.

the wt-NCI-H292 (+) cells (Fig. 6). Furthermore, silencing ADAM17 in MEF, using ADAM17 ${ }^{\text {ex/ex }}$ MEFs (-), and in NCI-H292 (-) cells decreased further the cell-cell interaction by about half in comparison to the control (Fig. 6). These results indicate that ADAM17 acts as an adhesion molecule and mediates fibroblast-carcinoma cell interaction. 


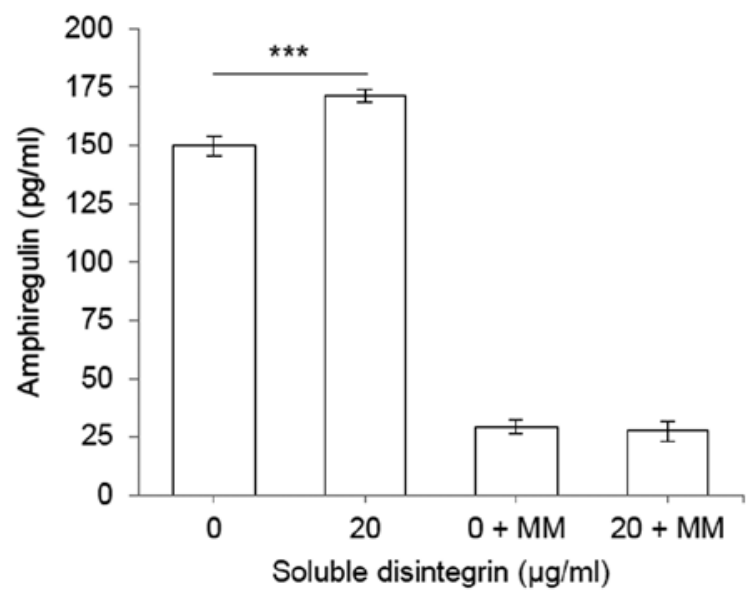

Figure 7. The soluble disintegrin domain of ADAM17 induces shedding of amphiregulin from MDA-MB-231 cells. MDA-MB-231 cells were incubated for $8 \mathrm{~h}$ in the absence $(0 \mu \mathrm{g} / \mathrm{ml})$ or presence of soluble disintegrin domain $(20 \mu \mathrm{g} / \mathrm{ml})$ and marimastat (MM) in serum-free medium. Afterwards supernatants were collected and amphiregulin was measured by enzyme-linked immunosorbent assay. Bars represent means \pm SD of three independent experiments. Asterisks indicate significant differences from the respective control values $\left({ }^{* * *} \mathrm{p}<0.0001\right)$

Soluble disintegrin domain of ADAM17 induces shedding of amphiregulin from $M D A-M B-231$ cells. The interaction between $\alpha 5 \beta 1$ integrin and ADAM17 keeps the enzyme in an inactive state $(9,10)$. This finding raises the assumption that the soluble disintegrin domain of ADAM17 should be able to induce shedding by competing with the wild-type molecule for the binding to $\alpha 5 \beta 1$ integrin. Thereby the complex between the integrin and enzyme should be dissolved, releasing an active ADAM17.

To test this hypothesis, we measured the shedding of amphiregulin in MDA-MB-231 cells incubated with or without the soluble disintegrin domain (Fig. 7). Amphiregulin, a substrate of ADAM17 (36), belongs to the epidermal growth factor family, and acts as mitogenic stimulator through binding to epidermal growth factor receptors (37). The resulting data showed that treatment of these cells with the soluble disintegrin domain increases shedding of amphiregulin in comparison to control cells treated with PBS. The shedding induced by soluble disintegrin could be inhibited by metalloprotease inhibitor, marimastat. Thus, soluble disintegrin domain binds to $\alpha 5 \beta 1$ integrin and consequently activates the wild-type molecule, most likely by releasing ADAM17 out of the inhibitory complex.

\section{Discussion}

The disintegrin domain of many ADAM family members has been shown to act as an adhesion ligand for integrins $(8,38)$. ADAM15 interacts with various integrins, including $\alpha \mathrm{v} \beta 3$-, $\alpha v \beta 1-, \alpha 9 \beta$ - and $\alpha 5 \beta 1$-integrin (39), while ADAM2 binds to $\alpha 6 \beta 1$ integrin, ADAM9 to $\alpha 6 \beta 1-, \alpha v \beta 5$ - and $\alpha 3 \beta 1$-integrin $(31,40)$ and ADAM28 to $\alpha 4 \beta 1$ integrin (8). In line with the work of Bax et al (7), we demonstrate that the recombinant soluble disintegrin domain of ADAM17 binds directly to the purified $\alpha 5 \beta 1$ integrin and is able to precipitate the
DN-ADAM17; confirming that the disintegrin domain of ADAM17 is a specific ligand for the $\alpha 5 \beta 1$ integrin and that the domain located C-terminally of the disintegrin domain of ADAM17 is not needed for this purpose. Additionally, the soluble disintegrin domain only is able to release ADAM17 out of the inhibitory complex, allowing the enzyme to shed its substrate amphiregulin.

In a next step, the adhesive property of ADAM17 at a cellular level was examined. MEF cells adhere strongly and dose-dependently to the immobilized disintegrin domain. Interestingly, carcinoma cells, which express high levels of ADAM17, are also able to adhere to the disintegrin domain. The adhesion of carcinoma cells to the immobilized disintegrin domain was inhibited by the soluble disintegrin domain, indicating a specific and direct interaction between carcinoma cells and ADAM17 via the disintegrin domain. These results demonstrate that fibroblasts as well as carcinoma cells adhere to ADAM17 via the $\alpha 5 \beta 1$ integrins, which is in good agreement with the data of Bax et al (7).

On the other hand, it has been shown that ADAM17 is upregulated in ovarian cancer $(22,23)$, colon carcinoma (24), lung cancer $(25,35)$, head and neck cancer (41) and its expression was associated with tumour invasion and metastasis $(21,42)$. In these studies, the authors correlated metastasis and shedding of different growth factors induced by upregulation of ADAM17, but did not further analyze the resulting enhancement of adhesive properties of cancer cells to fibroblasts. For that purpose, cell-cell interaction assays between fibroblasts and carcinoma cells in the presence and absence of the soluble disintegrin domain of ADAM17 were performed. The soluble disintegrin domain reduces the fibroblast-carcinoma cell interaction, indicating that ADAM17 mediates directly the interaction between fibroblasts and carcinoma cells.

Similar effects have been shown for ADAM9 (31). The disintegrin domain of ADAM9 mediates a direct interaction between melanoma cells and fibroblasts and ADAM9 improves the invasive ability of melanoma cells (31). The same observation has been made in case of ADAM17 where silencing of ADAM17 expression in NCI-H292 cells leads to a high reduction in their ability to migrate $(27,43)$. Therefore, ADAM17 mediated shedding of growth factor and adhesion molecules seem not to be the only tumour promoting factor of the metalloprotease. In addition, ADAM17 mediates the interaction between fibroblasts and carcinoma cells via its disintegrin domain, which seems to be an important factor for carcinoma invasion. The ADAM17/ $\alpha 5 \beta 1$ integrin complex keeps the protease in an inactive state. Release of the ADAM17 from this complex leads to an active $\operatorname{ADAM} 17(9,10)$, which then is able to shed membrane-bound growth factors, like amphiregulin, resulting in tumour growth. Therefore, ADAM17 promotes migration as well as proliferation of tumour cells. The combined action of this metalloprotease makes ADAM17 an even more interesting target for cancer therapy.

\section{Acknowledgements}

This study was supported by the Deutsche Forschungsgemeinschaft (SFB 877, A6, Z3) and the cluster of excellence 'Inflammation at Interfaces'. 


\section{References}

1. Brocker CN, Vasiliou V and Nebert DW: Evolutionary divergence and functions of the ADAM and ADAMTS gene families. Hum Genomics 4: 43-55, 2009.

2. White JM: ADAMs: modulators of cell-cell and cell-matrix interactions. Curr Opin Cell Biol 15: 598-606, 2003.

3. Takeda S: Three-dimensional domain architecture of the ADAM family proteinases. Semin Cell Dev Biol 20: 146-152, 2009.

4. Liu H, Shim AH and He X: Structural characterization of the ectodomain of a disintegrin and metalloproteinase-22 (ADAM22), a neural adhesion receptor instead of metalloproteinase: insights on ADAM function. J Biol Chem 284 29077-29086, 2009.

5. Janes PW, Saha N, Barton WA, et al: Adam meets Eph: an ADAM substrate recognition module acts as a molecular switch for ephrin cleavage in trans. Cell 123: 291-304, 2005.

6. Reiss K and Saftig P: The 'a disintegrin and metalloprotease' (ADAM) family of sheddases: physiological and cellular functions. Semin Cell Dev Biol 20: 126-137, 2009.

7. Bax DV, Messent AJ, Tart J, et al: Integrin alpha5beta1 and ADAM-17 interact in vitro and co-localize in migrating HeLa cells. J Biol Chem 279: 22377-22386, 2004.

8. McGinn OJ, English WR, Roberts S, Ager A, Newham P and Murphy G: Modulation of integrin alpha4beta1 by ADAM28 promotes lymphocyte adhesion and transendothelial migration. Cell Biol Int 35: 1043-1053, 2011.

9. Saha A, Backert S, Hammond CE, Gooz M and Smolka AJ: Helicobacter pylori CagL activates ADAM17 to induce repression of the gastric H, K-ATPase alpha subunit. Gastroenterology 139: 239-248, 2010 .

10. Gooz P, Dang Y, Higashiyama S, Twal WO, Haycraft CJ and Gooz M: A disintegrin and metalloenzyme (ADAM) 17 activation is regulated by alpha5betal integrin in kidney mesangial cells. PLoS One 7: e33350, 2012

11. Milla ME, Leesnitzer MA, Moss ML, et al: Specific sequence elements are required for the expression of functional tumor necrosis factor-alpha-converting enzyme (TACE). J Biol Chem 274: 30563-30570, 1999.

12. Lorenzen I, Trad A and Grötzinger J: Multimerisation of A disintegrin and metalloprotease protein-17 (ADAM17) is mediated by its EGF-like domain. Biochem Biophys Res Commun 415: 330-336, 2011.

13. Reddy P, Slack JL, Davis R, et al: Functional analysis of the domain structure of tumor necrosis factor-alpha converting enzyme. J Biol Chem 275: 14608-14614, 2000.

14. Tape CJ, Willems SH, Dombernowsky SL, et al: Cross-domain inhibition of TACE ectodomain. Proc Natl Acad Sci USA 108 5578-5583, 2011.

15. Lorenzen I, Lokau J, Düsterhöft S, et al: The membrane-proximal domain of A Disintegrin and Metalloprotease 17 (ADAM17) is responsible for recognition of the interleukin- 6 receptor and interleukin-1 receptor II. FEBS Lett 586: 1093-1100, 2012.

16. Smith KM, Gaultier A, Cousin H, Alfandari D, White JM and DeSimone DW: The cysteine-rich domain regulates ADAM protease function in vivo. J Cell Biol 159: 893-902, 2002.

17. Soond SM,Everson B, Riches DW and Murphy G: ERK-mediated phosphorylation of Thr735 in TNFalpha-converting enzyme and its potential role in TACE protein trafficking. J Cell Sci 118: 2371-2380, 2005

18. Edwards DR, Handsley MM and Pennington CJ: The ADAM metalloproteinases. Mol Aspects Med 29: 258-289, 2008.

19. Kommaddi RP, Thomas R, Ceni C, Daigneault K and Barker PA: Trk-dependent ADAM17 activation facilitates neurotrophin survival signaling. FASEB J 25: 2061-2070, 2011.

20. McGowan PM, McKiernan E, Bolster F, et al: ADAM-17 predicts adverse outcome in patients with breast cancer. Ann Oncol 19: 1075-1081, 2008.

21. McGowan PM, Ryan BM, Hill AD, McDermott E, O'Higgins N and Duffy MJ: ADAM-17 expression in breast cancer correlates with variables of tumor progression. Clin Cancer Res 13: 2335-2343, 2007.

22. Tanaka Y, Miyamoto S, Suzuki SO, et al: Clinical significance of heparin-binding epidermal growth factor-like growth factor and a disintegrin and metalloprotease 17 expression in human ovarian cancer. Clin Cancer Res 11: 4783-4792, 2005.
23. Rosso O, Piazza T, Bongarzone I, et al: The ALCAM shedding by the metalloprotease ADAM17/TACE is involved in motility of ovarian carcinoma cells. Mol Cancer Res 5: 1246-1253, 2007.

24. Blanchot-Jossic F, Jarry A, Masson D, et al: Up-regulated expression of ADAM17 in human colon carcinoma: co-expression with EGFR in neoplastic and endothelial cells. J Pathol 207: 156-163, 2005.

25. Dijkstra A, Postma DS, Noordhoek JA, et al: Expression of ADAMs ('a disintegrin and metalloprotease') in the human lung. Virchows Arch 454: 441-449, 2009.

26. Badoual C, Bouchaud G, Agueznay Nel H, et al: The soluble alpha chain of interleukin-15 receptor: a proinflammatory molecule associated with tumor progression in head and neck cancer. Cancer Res 68: 3907-3914, 2008.

27. Zheng X, Jiang F, Katakowski M, Zhang ZG, Lu QE and Chopp M: ADAM17 promotes breast cancer cell malignant phenotype through EGFR-PI3K-AKT activation. Cancer Biol Ther 8: 1045-1054, 2009.

28. Trad A, Hedemann N, Shomali M, Pawlak V, Grotzinger J and Lorenzen I: Development of sandwich ELISA for detection and quantification of human and murine a disintegrin and metalloproteinase 17. J Immunol Methods 371: 91-96, 2011.

29. Trad A, Hansen HP, Shomali M, et al: ADAM17-overexpressing breast cancer cells selectively targeted by antibody-toxin conjugates. Cancer Immunol Immunother: Sep 1, 2012 (Epub ahead of print).

30. Yamamoto K, Trad A, Baumgart A, et al: A novel bispecific single-chain antibody for ADAM17 and CD3 induces T-cellmediated lysis of prostate cancer cells. Biochem J 445: 135-144, 2012.

31. Zigrino $\mathrm{P}, \mathrm{Nischt} \mathrm{R}$ and Mauch $\mathrm{C}$ : The disintegrin-like and cysteine-rich domains of ADAM-9 mediate interactions between melanoma cells and fibroblasts. J Biol Chem 286: 6801-6807, 2011

32. Chalaris A, Rabe B, Paliga K, et al: Apoptosis is a natural stimulus of IL6R shedding and contributes to the proinflammatory trans-signaling function of neutrophils. Blood 110: 1748-1755, 2007.

33. Jovic M, Naslavsky N, Rapaport D, Horowitz M and Caplan S: EHD1 regulates beta1 integrin endosomal transport: effects on focal adhesions, cell spreading and migration. J Cell Sci 120: 802-814, 2007.

34. Chalaris A, Adam N, Sina C, et al: Critical role of the disintegrin metalloprotease ADAM17 for intestinal inflammation and regeneration in mice. J Exp Med 207: 1617-1624, 2010.

35. Baumgart A, Seidl S, Vlachou P, et al: ADAM17 regulates epidermal growth factor receptor expression through the activation of Notch1 in non-small cell lung cancer. Cancer Res 70: 5368-5378, 2010

36. Sternlicht MD and Sunnarborg SW: The ADAM17amphiregulin-EGFR axis in mammary development and cancer. J Mammary Gland Biol Neoplasia 13: 181-194, 2008.

37. Kuramochi H, Nakajima G, Kaneko Y, et al: Amphiregulin and Epiregulin mRNA expression in primary colorectal cancer and corresponding liver metastases. BMC Cancer 12: 88, 2012.

38. Toquet C, Colson A, Jarry A, et al: ADAM15 to alpha5beta1 integrin switch in colon carcinoma cells: A late event in cancer progression associated with tumor dedifferentiation and poor prognosis. Int J Cancer 130: 278-287, 2012.

39. Lucas $\mathrm{N}$ and Day ML: The role of the disintegrin metalloproteinase ADAM15 in prostate cancer progression. J Cell Biochem 106: 967-974, 2009.

40. Desiderio UV, Zhu X and Evans JP: ADAM2 interactions with mouse eggs and cell lines expressing alpha4/alpha9 (ITGA4/ITGA9) integrins: implications for integrin-based adhesion and fertilization. PLoS One 5: e13744, 2010.

41. Kornfeld JW, Meder S, Wohlberg M, et al: Overexpression of TACE and TIMP3 mRNA in head and neck cancer: association with tumour development and progression. Br J Cancer 104: 138-145, 2011.

42. Gooz M: ADAM-17: the enzyme that does it all. Crit Rev Biochem Mol Biol 45: 146-169, 2010.

43. Zheng X, Jiang F, Katakowski M, Lu Y and Chopp M: ADAM17 promotes glioma cell malignant phenotype. Mol Carcinog 51: $150-164,2012$. 